\title{
Combined transhepatic and endoscopic procedures in the biliary system
}

\author{
Anthony G. Verstandig, Eran Goldin ${ }^{1}$, Talia Sasson, Gerald Weinberger, \\ Dov Wengrower ${ }^{1}$, Alex Fich ${ }^{1}$ and Emeric Lax
}

Departments of Radiology and ${ }^{1}$ Gastroenterology, Hadassah University Hospital, POB 12000 Ein Kerem, Jerusalem 91120, Israel

Summary: Endoscopic biliary procedures are 89-97\% successful in skilled hands. The commonest causes of failure are inability to cannulate the papilla of Vater due to difficult anatomy or tortuosity of the distal common bile duct and failure to cross a rigid biliary stricture. In nearly all of these cases, successful endoscopic procedures can be completed after percutaneous antegrade placement of a small catheter or guidewire to the duodenum. In 44 such combined procedures on 42 patients, the success rate was 43 (98\%). There were two severe and eight mild complications. Combined procedures overcome the difficulties caused by tortuous biliary ducts and rigid strictures while obviating the need for more extensive percutaneous procedures and transhepatic tract dilatation.

\section{Introduction}

Endoscopy is recognized as a valuable technique for performing most interventional procedures in the extrahepatic biliary tree. ${ }^{1-3}$ However, even in skilled hands, technical factors can prevent cannulation of the papilla of Vater, passage through a biliary stricture, or placement of a stent., 4 These difficulties can nearly always be overcome by percutaneous antegrade placement of a guidewire or small catheter through the biliary system into the duodenum prior to repeat endoscopy. ${ }^{6-12}$ This paper documents our experience with this combined approach.

\section{Materials and methods}

Between June 1986 and June 1992, 1,558 endoscopic biliary procedures were performed at our institution. A total of 730 of these were therapeutic, comprising 459 stent insertions, 427 for malignant and 32 for benign disease and 271 stone removal procedures. A total of 44 percutaneous procedures were performed on 42 patients to facilitate subsequent endoscopic therapy. All of the radiographs, in-patient charts and endoscopy reports of these patients were reviewed.

There were 20 women and 22 men aged from 35 to 81 years (mean age 61). The indications for biliary intervention are listed in Table I. Surgically

Correspondence: A.G. Verstandig, M.B., B.S.

Accepted: 16 November 1992
Table I

Indication for biliary intervention No. 흐

Pancreatic carcinoma

Common duct stones

Metastatic porta hepatis nodes

Cholangiocarcinoma (porta hepatis 4 CBD 1)

Sclerosing cholangitis*

Post cholecystectomy stricture with bile leak

Post cholecystectomy occlusion with fistula to duodenum

Total patients

*One case primary, the other secondary to hepatic artery infusion chemotherapy. $\mathrm{CBD}=$ common bile duct.

placed T-tubes were present in seven patients, three with pancreatic carcinoma, three with common bile duct stones and in the case of a patient with $N_{\mathscr{S}}$ sclerosing cholangitis.

The causes for failure of the initial endoscopic procedures are listed in Table II.

\section{Techniques}

In the seven patients with T-tubes, a torquable $\frac{T}{0}$ Ring Lunderquist biliary wire (Cook Inc., Bloom- $\frac{\overrightarrow{0}}{\mathbb{D}}$ ington, IN, USA) was passed through the inferior $\frac{\text { }}{\Phi}$ limb of the tube into the duodenum. A straighto 
Table II Causes of failed endoscopic sphincterotomy or retrograde stenting

\begin{tabular}{lr}
\hline & No. \\
\hline Inability to cannulate papilla & 25 \\
Inability to cross stricture & 7 \\
Extrinsic mass deforming duodenum & 2 \\
Passage prevented by T-tube & 2 \\
Unsuccessful endoscopic stent change & 2 \\
Passage prevented by stones & 3 \\
Billroth 2 gastroenterostomy & 2 \\
Inability to cannulate left duct selectively & 1 \\
Total procedures & 44 \\
\hline
\end{tabular}

angiographic catheter was then placed over the guidewire into the duodenum.

In the remaining 35 patients, 37 percutaneous transhepatic biliary drainage procedures were performed using standard technique. ${ }^{13}$ All patients received prophylactic antibiotics and had normal coagulation profiles. Whenever possible, the stricture or stones were crossed at the initial session and a 5-8 French straight polyethylene catheter (Cook Inc.) placed through the Papilla of Vater with $1-2 \mathrm{~cm}$ projecting into the duodenum and multiple side holes in the bile ducts. The biliary system was drained externally until subsequent endoscopy. In eight patients the stricture could not be crossed at the initial procedure and placement of a catheter into the duodenum was achieved after a period of external biliary drainage. In one patient, initial biliary decompression was achieved by percutaneous cholecystostomy. Percutaneous transhepatic biliary drainage was performed after failure to cannulate the cystic duct.

In 32 patients, 34 procedures were performed to cannulate the right biliary system. In three patients left biliary drainage was performed including two who had undergone right hepatectomy for metastatic disease. The third patient had a cholangiocarcinoma at the porta hepatis (Klatskin tumour) with persistent left duct dilatation and cholangitis after successful endoscopic stenting of his isolated right biliary system.

For logistical reasons endoscopy usually could not be performed immediately after percutaneous transhepatic biliary drainage or T-tube tract cannulation. After an interval of $0-13$ days (mean 2.7) endoscopic access to the biliary system was obtained by one of three methods: (1) In 12 procedures the endoscopic wire could be passed alongside the percutaneous catheter into the biliary system (Figure 1). The antegrade catheter both indicates the position of the ampulla and straightens the distal common duct facilitating retrograde catheterization. (2) In 15 procedures the endoscopic



Figure 1 Endoscopic stone extraction. The 6 French percutaneous catheter (arrows) acts as a guide alongside which the sphincterotome and subsequently the basket could be passed.

guidewire was passed into the lumen of the percutaneous catheter which was then withdrawn into a peripheral duct to allow stent placement (Figure 2a). In some of these cases, the wire was passed out of the end of the percutaneous catheter and clamped outside the body to facilitate stent placement through a rigid stenosis (Figure 2b). (3) In the remaining 16 cases in which these techniques were unsuccessful, a $300 \mathrm{~cm}$ or longer (ideally $400 \mathrm{~cm}$ ) 0.038 inch diameter standard floppy tipped wire (Cook Inc.) was passed antegrade through the percutaneous catheter into the duodenum and its end grasped by a stone basket introduced through the endoscope. The wire was then withdrawn through the endoscope by traction on the basket. In both the second technique and in this one, the presence of a guidewire, which could be controlled at both ends, usually allowed easy placement of stents. In five cases in which there was difficulty advancing the stent through rigid strictures, the stent pusher, catheter and wire were clamped together as a single unit at the endoscopic end, and the stent advanced to the required position by traction on the wire at the percutaneous site.

A total of 39 stents were placed in 37 patients. In all cases, final positioning of the stent was moni- 

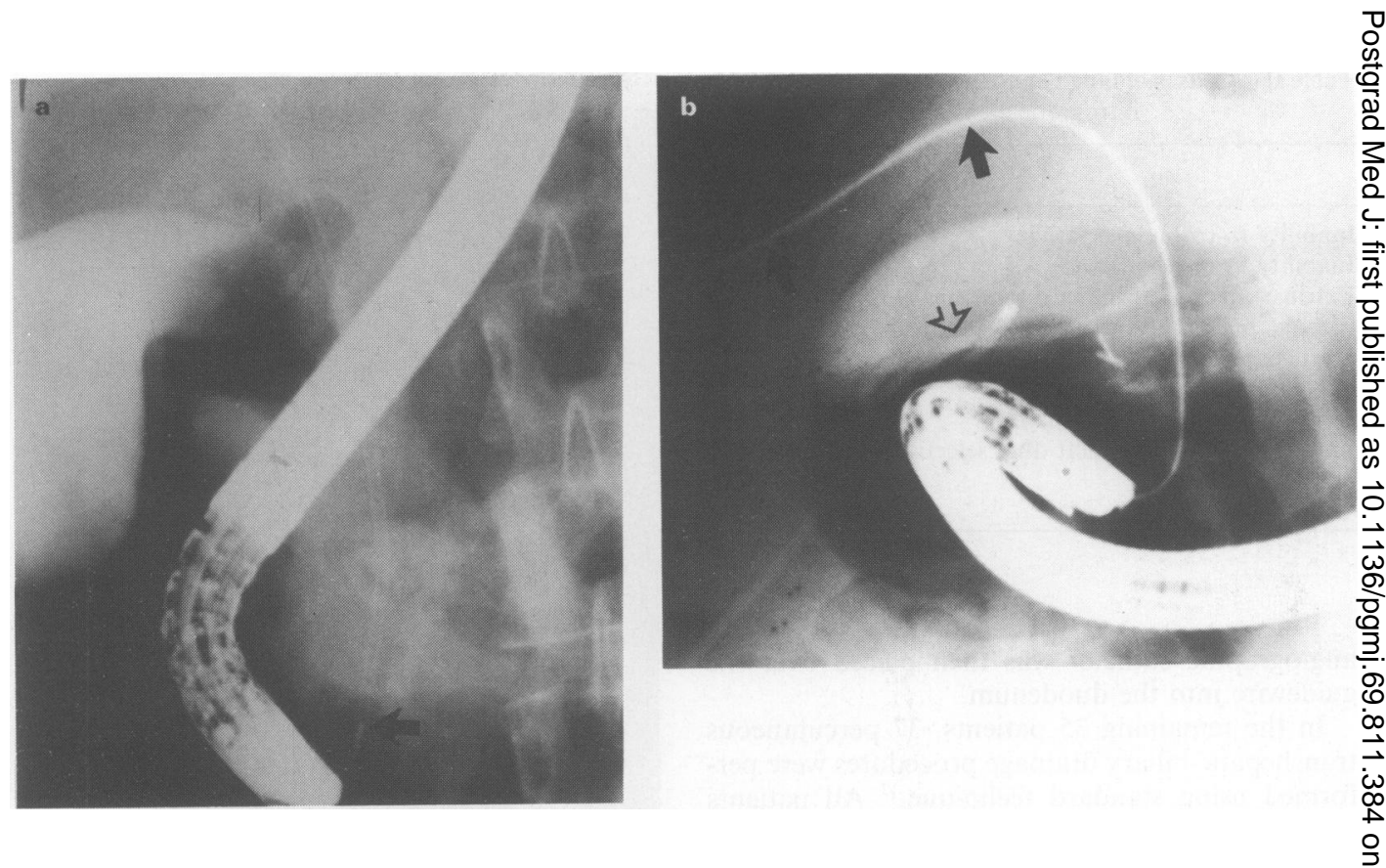

Figure 2 (a) Percutaneous catheter being cannulated by the endoscopic guidewire (arrow). (b) In a different patient, $\longrightarrow$ the endoscopic wire (arrows) has been advanced to the percutaneous entry site where it can be clamped to allow the $z$ stent to be pushed through a rigid stricture. There is a drainage catheter in the gallbladder (hollow arrow).

tored fluoroscopically with reference to films taken during previous antegrade or T-tube cholangiography. When the stent position was deemed satisfactory, the guidewire was withdrawn and where possible antegrade cholangiography was performed to determine the position of the proximal end of the stent and to confirm free drainage to the duodenum (Figure 3). The percutaneous catheter was then removed. Stents were placed in three of the patients with common bile duct stones. In the other four patients, percutaneous transhepatic biliary drainage facilitated subsequent endoscopic sphincterotomy and stone basketing.

\section{Results and complications}

Endoscopic choledocholithotomy was successful with no complications in the four patients in whom it was attempted. Initial satisfactory stent placement was achieved in 38 out of 39 procedures. In one patient the stent was too short resulting in inadequate biliary drainage and persistent jaundice. After 3 days, the stent was replaced endoscopically by a longer one without recourse to repeat percutaneous transhepatic biliary drainage.

Short-term follow-up was available on 35 patients who had undergone 37 procedures. There were ten periprocedural complications, eight mildō and two severe. Five patients had episodes of cholangitis with fever of more than $38^{\circ} \mathrm{C}$, three after percutaneous drainage, one after stent inser- $\overrightarrow{\vec{O}}$ tion and one after a single session combined 3 procedure. All these episodes resolved with continued antibiotic and supportive treatment. One patient had transient pancreatitis with fever,을 abdominal pain and a serum amylase of 1,370 units $\frac{3}{-}$. after stent insertion. Three patients had bleeding complications. Two had self-limiting haemobilia. post percutaneous drainage not requiring trans- $O$ fusion. The third patient had a self-limiting bleed after a single session combined procedure requiring 음 transfusion of 2 units of blood but no other $>$ treatment. There was one procedure-related death from septic shock in a 76 year old patient with an obstructing pancreatic carcinoma and grossly infected bile.

\section{Discussion}

In skilled hands, endoscopic biliary procedures are $89-97 \%$ successful. ${ }^{1,4,7}$ The cause of failure is ${ }^{7}$ usually inability to cannulate the papilla of Vater $\frac{\text { Oे }}{0}$ even after using the precutting technique, or $\stackrel{?}{\mathscr{Q}}$ inability to cross a tortuous, rigid ductal stricture. $\stackrel{\unrhd}{\varrho}$ 


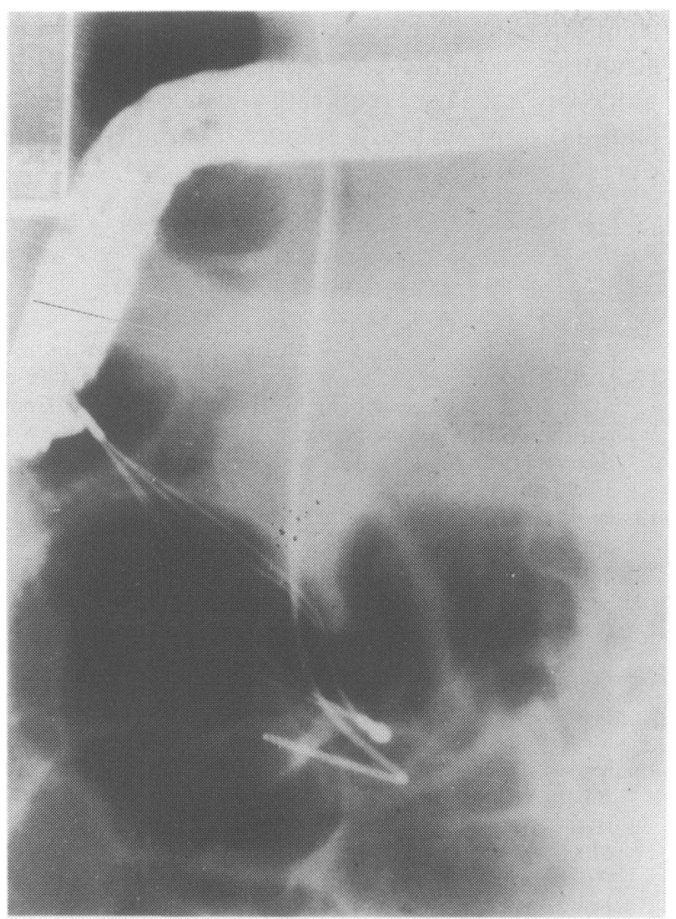

Figure 3 Final antegrade cholangiography (in this case via a cholecystostomy catheter) demonstrating good stent position and free flow of contrast to the duodenum.

Other causes of failed endoscopic procedures are listed in Table I. These can nearly all be overcome by the presence of a small catheter or guidewire placed antegrade through the papilla followed by endoscopic catheterization using one of the techniques described above. The long tortuous route through the working channel of the endoscope hampers fine control of the catheter and guidewire whereas the much shorter transhepatic route allows successful negotiation of tortuous bile ducts and ductal strictures.

Use of this combined approach was first described in 1980 using the transhepatic route ${ }^{14}$ and via an existing cholecystostomy. ${ }^{15}$ Since then there have been several reports of its use to facilitate endoscopic sphincterotomy ${ }^{6-8}$ and stenting. ${ }^{10,12,23}$ Kerlan and Ring reported a combined transhepatic and peroral approach which does not require endoscopy. ${ }^{16}$

Endoscopic stenting of bile duct strictures has a lower morbidity and mortality and a higher rate of successful biliary drainage than percutaneous biliary endoprosthesis placement. ${ }^{1}$ In addition Hall et al. reported a higher rate of successful endoscopic stent replacement with endoscopically placed stents compared with percutaneously placed endo- prostheses. ${ }^{12}$ The higher complication rate of transhepatic procedures is mainly due to the trauma of creating a tract through the liver parenchyma and dilating it to $10-14$ French. Combined procedures requiring tract dilatation to only 5-8 French may well have a lower complication rate as reported in the comparative study of Hall although the results were not statistically significant. ${ }^{12}$ In this series the complication rate is comparable to that reported with percutaneous biliary stenting or drainage. . $^{1,17,18}$ There are, however, definite advantages to the combined approach. The avoidance of dilating the transhepatic tract to $10 \mathrm{~F}$ or more makes the procedure far less traumatic and acceptable for the patient, and larger series may eventually show a lower complication rate. The presence of external drainage tubes with local entrance site complications and adverse psychological effects is avoided. In addition, the use of percutaneous prostheses which may be difficult to replace endoscopically is avoided. In patients with large common bile duct stones in whom failure of endoscopic therapy means probable surgery, the advantages of lithotomy or stenting by the combined approach are obvious. The shorter percutaneous procedure also results in a lower radiation dose to the radiologist.

Biliary obstruction at the confluence of the main intrahepatic biliary ducts presents a special problem. One patient required a combined left-sided procedure to supplement successful endoscopic stenting of the right biliary system. A second patient was successfully stented by the combined approach but after failure of stent replacement due to a sharp angle at the site of obstruction was managed by percutaneous drainage. Since patients with Klatskin tumours may survive for a long time, placing a stent that is difficult or impossible to replace endoscopically is not recommended. For this reason, after our initial experience, subsequent patients with Klatskin tumours (not included in this series) in whom endoscopic stenting was unsuccessful were managed by percutaneous drainage. Using this combined approach, the success rate of endoscopic procedures at our institution has risen from $93.5 \%$ to $99.5 \%$.

Chespak et al.$^{19}$ recently reported an increased success rate of endoscopic interventions using the highly controllable Glidewire (Terumo, Meditech Watertown, MA, USA) through the endoscope. This technique may obviate the need for some combined procedures in patients with intrahepatic duct lesions or very tortuous non-rigid strictures.

The recent introduction of percutaneous expandible metallic biliary stents allowing the introduction of large calibre stents $(8-10 \mathrm{~mm})$ through 7-12 French sheaths ${ }^{20-21}$ provides an alternative means of percutaneous stent insertion in some cases of failed endoscopic stenting. Despite their large internal diameter, these expensive devices 
have not yet demonstrated a higher long-term patency rate compared to standard percutaneous or endoscopic stents. ${ }^{21,22}$ Further evaluation is required to determine their place in the treatment of biliary obstruction. Even if problems of longterm patency and tumour ingrowth through the

\section{References}

1. Speer, A.G., Cotton, P.B., Russel, R.C.G. et al. Randomised trial of endoscopic versus percutaneous stent insertion in malignant obstructive jaundice. Lancet 1987, ii: 57-62.

2. Mee, A.S., Vallon, A.G., Croker, J.R. \& Cotton, P.B. Non-operative removal of bile duct stones by duodenoscopic sphincterotomy in the elderly. Br Med J 1981, 2: 521-523.

3. Brambs, J.H., Billman, P., Pausch, J. et al. Non-surgical biliary drainage: endoscopic conversion of percutaneous transhepatic into endoprosthetic drainage. Endoscopy 1986, 18: $52-54$.

4. Cremer, M. Complications of endoscopic spincterotomy. In: Classen, M., Geenen, J. \& Kawai, K. (eds) The Papilla Vateria and its Diseases. Verlag Gerhard Witzstrock, New York, 1979.

5. Marks, W.M., Freeny, P.C., Ball, T.J. \& Gannan, R.M Endoscopic retrograde biliary drainage. Radiology 1984, 152: 357-360.

6. Long, W.B., Schwartz, W. \& Ring, E.J. Endoscopic sphincterotomy assisted by antegrade catheterisation. Gastrointestinal Endosc 1984, 30: 36-39.

7. Shovron, P.J., Cotton, P.B., Mason, R.R. et al. Percutaneous transhepatic assistance for duodenoscopic sphincterotomy. Gut 1985, 26: 1373-1376.

8. Tanaka, M., Matsumoto, S., Ikeda, S. et al. Endoscopic sphincterotomy in patients with difficult cannulation: use of antegrade guide. Endoscopy 1986, 18: 87-89.

9. Foutch, P.G,. Chinichian, A., Talbert, G. \& Sanowski, R.A Endoscopic conversion of an external biliary drain to an internal common bile duct stent. Gastrointest Endosc 1987, 33: 379-381.

10. Robertson, D.A.F., Ayres, R., Hacking, C.N. et al. Experience with a combined percutaneous and endoscopic approach to stent insertion in malignant obstructive jaundice. Lancet 1987, ii: 1449-1452.

11. Ayela, P., Ponchon, T., Valette, P.J. et al. A combined endoscopic and radiological method for biliary drainage. Gastrointest Endosc 1988, 34: 42-44. stent interstices are overcome, the stenting of rigi strictures requiring control of both ends of the guidewire and cases requiring endoscopic choledo 3 cholithotomy will still require combined proce 2 dures if initial endoscopy fails.

12. Hall, R.I., Denyer, M.E. \& Chapman, A.H. Palliation of obstructive jaundice with a biliary endoprosthesis. Comes parison of insertion by the percutaneous-transhepatic and the combined percutaneous-endoscopic routes. Clin Radiof 1989, 40: $186-189$.

13. Ring, E.J., Oleaga, J.A., Freiman, D.B. et al. Therapeutifu applications of catheter cholangiography. Radiology 1978 128: 333-338.

14. Nimura, Y., Miyata, K., Yasui, K. et al. Percutaneou疋. transhepatic papillotomy. Gastroenterol Endosc 1980, 220 36-39.

15. Mason, R.R. \& Cotton, P.B. Combined endoscopic and゚ percutaneous trans-cystic approach to a retained common duct stone. Br J Radiol 1980, 53: 38-39.

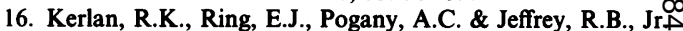
Biliary endoprosthesis: insertion using a combined peroralo transhepatic method. Radiology 1984, 150: 828-830.

17. Mueller, P.R., van Sonnenberg, E. \& Ferrucci, J.T., Jr $\overrightarrow{-}$ Percutaneous biliary drainage: technical and catheter relateo problems in 200 procedures. AJR 1982, 138: 17-23.

18. Carrasco, C.H., Zornoza, J. \& Bechtel, W.J. Malignant biliary obstruction: complications of percutaneous drainfsece Radiology 1984, 152: 343-346.

19. Chespak, L.W., Ring, E.J., Shapiro, H.A. et al. Multifis ciplinary approach to complex endoscopic biliary interfing tion. Radiology 1989, 170: 995-997.

20. Coons, H.G. Self expanding stainless steel biliary stentsō Radiology 1989, 170: 979-983.

21. Irving, J.D., Adam, A., Dick, R. et al. Gianturco expandablēD metallic biliary stents: Results of a European clinical trial Radiology 1989, 172: 321-326.

22. Yoshioko, T., Sakaguchi, H., Yoshimura, H. et al. Expan dable metallic biliary endoprosthesis: Preliminary clinicab evaluation. Radiology 1990, 177: 253-257.

23. Tat-Kin, T., Crampton, A.R., Bernstein, J.R et al. Percutaneous-endoscopic biliary stenting in patients with occ음 luded surgical bypass. Am J Med 1990, 88: 343-348. 


\section{Errata}

Re: Postgraduate Medical Journal 1993, 69: Re: Postgraduate Medical Journal 1993, 69: 384-388.

Combined transhepatic and endoscopic proce100-106.

dures in the biliary system: A.G. Verstandig, E. Goldin, T. Sasson, G. Weinberger, D. Wengower, A. Fich and E. Lax

We apologise for printing the incorrect illustration for Figure 3 of this paper. The correct figure with legend is reproduced below.

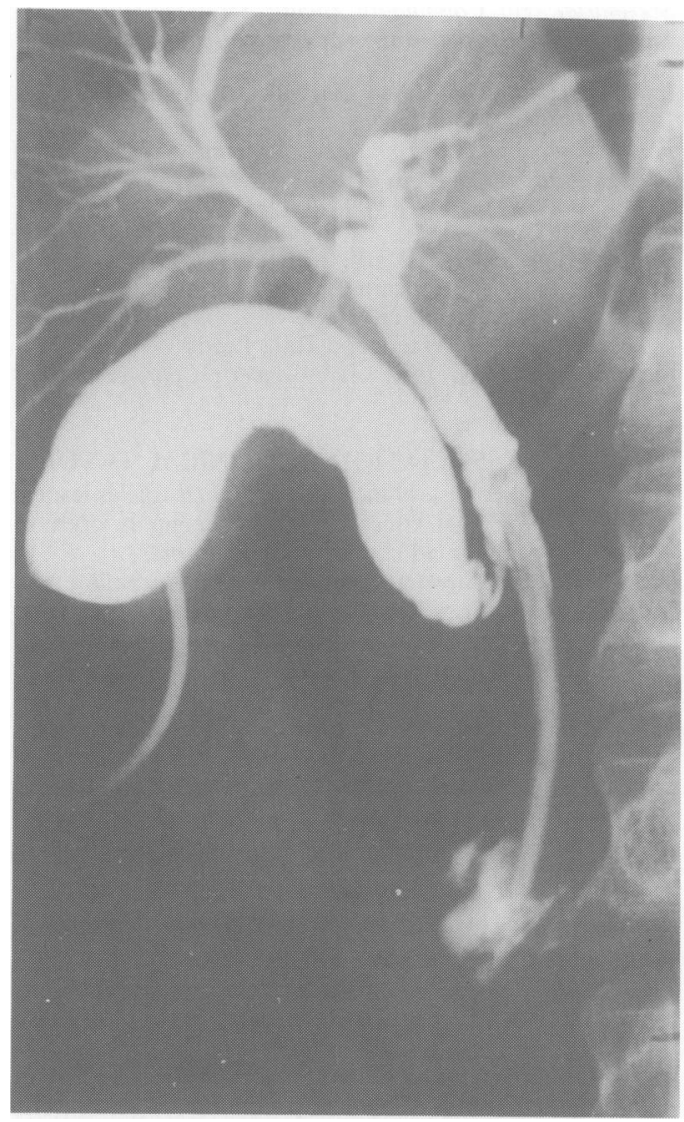

Relationship between smoking, weight and attitudes to weight in adolescent schoolgirls: C. Halek, S. Kerry, H. Humphrey, A.H. Crisp and J.M. Hughes.

An author's error occurred in this paper. The statistical test used to compare menarchal age was an $F$ test not a chi-square test as reported. The $P$-values are correct.

Figure 3 Final antegrade cholangiography (in this case via a cholecystostomy catheter) demonstrating good stent position and free flow of contrast to the duodenum. 